\title{
Phytochemicals in medicine and food
}

\author{
Jianbo Xiao
}

Published online: 2 April 2015

(C) Springer Science+Business Media Dordrecht 2015

From June 26th to 29th of 2015, the Phytochemical Society of Europe (PSE) meeting in collaboration with the Phytochemical Society of Asia (PSA)-International Symposium on Phytochemicals in Medicine and Food (ISPMF2015) will be held in Shanghai, China. It is the first time to organize a PSE-PSA conference in China, jointly organized by Macau University of Science and Technology and Shanghai Normal University. The local organizing and sponsoring institutions contain Beijing Normal University, Yancheng Institute of Technology, Guiyang Medical University, and Fudan University. Over 230 scientists from 50 counties have registered to attend this conference.

The international organizing committee of ISPMF2015 consists of Prof. Simon Gibbons (UK), Prof. Miroslav Strnad (Czech), Dr. Milen I. Georgiev (Bulgaria), Dr. Krystyna Skalicka-Woźniak (Poland), Dr. Amir Reza Jassbi (Iran), Prof. Franz Bucar (Austria), Prof. Satya Sarker (UK), Dr. Jianbo Xiao

J. Xiao ( $\square)$

State Key Laboratory of Quality Research in Chinese Medicine, Macau University of Science and Technology, Taipa, Macau

e-mail: jianboxiao@yahoo.com

\section{J. Xiao}

Institut für Pharmazie und Lebensmittelchemie, Universität Würzburg, Am Hubland, 97074 Würzburg, Germany
(China \& Germany, Chairman), and Prof. Zhihong Jiang (Macau, Co-Chairman). The international scientific advisory board is comprised of the representatives from Germany, UK, Italy, Romania, Turkey, Netherlands, China, Pakistan, Botswana, Japan, Canada, Australia and USA. The international organizing committee and scientific advisory board of ISPMF2015 assembled an exciting and diverse program, featuring 10 keynote lectures, 24 invited lectures, 50 short lectures, and about 130 posters, which dedicate to creating a stage for exchanging the latest research results in the phytochemicals for food and human health. The overall objective is to provide a forum for the exchange of wide information on natural bioactive compounds from plants and other natural sources used for medicine and food.

ISPMF2015 also has obtained the supports form several international journals including Phytochemistry Reviews (Springer), Food Chemistry (Elsevier), Critical Reviews in Food Science and Nutrition (Taylor \& Francis), Nutrients (MDPI), Current Pharmaceutical Biotechnology (Bentham), Comprehensive Reviews in Food Science and Food Safety (Wiley), and Journal of Agricultural and Food Chemistry (ACS).

Scientists have brought an interesting trend in pharmaceutical development since the begin of twenty first century: return to nature as a source of potential drugs (Georgiev 2013; Lanzotti 2014). Various nature-origin phytochemicals, such as polyphenols, 
flavonoids, alkaloids, polysaccharides, stilbenoids, and essential oils, have received an increased attention due to their considerable biological benefits (Georgiev 2014; Xiao et al. 2014). Evidence based on epidemiological data have showed that the natural bioactive compounds play an important role in preventing and managing of modern diseases such as cancers, diabetes, Alzheimer's diseases and cardiovascular diseases (Andrae-Marobela et al. 2013; Xiao and Shao 2013; Lanzotti and Xiao 2014; Xiao 2015; Xiao and Jiang 2015).

Thymus species have been widely used as the medicinal or aromatic herbs in the pharmaceutical and food industries. Zeljković and Maksimović focus on the chemical composition and bioactivity of essential oils from Thymus species in Balkan Peninsula (Zeljković and Maksimović, 2015). The authors found that more than $90 \%$ of investigations according to the available literature emphasized on the antimicrobial activities of Thymus essential oils. There are still missing data for bioactivities for most investigated plants.

Naturally occurring peptides isolated from buckwheat seeds are prospectively applied in the function food and traditional Chinese medicine. Zhou et al. (2015) reviewed the bioactivities including the antibacterial, inhibition of trypsin, antitumor, hypocholesterol, hypotensive and antidiabetic effects of buckwheat proteins and their enzymatic hydrolysates. The antitumor and trypsin inhibitory activities are related to the special active site of the peptides, while the hypolipidemic and the hypotensive capacities are associated with the unique amino acids composition of buckwheat proteins.

Calceolaria spp is regarded as a notorious weed and a popular ornamental garden plant, which has also been used as medicinal herb. Cespedes et al. (2015) summarized the phytochemistry and bioativities of several Calceolaria species. New iridoids, flavonoids and phenylpropanoids for these species have been isolated, identified and tested for their insecticidal, antimicrobial, anticancer, proteinase, tyrosinase, and acetylcholinesterase inhibitory activities. Calceolaria flavonoids have been found to be potent insecticides and fungicides phenylpropanoids mixtures and iridoids was found to be antifeedant. Kaempferol and gallic acid showed a strong fungicidal activity against phytopathogenic strains. Naphthoquinones have anticancer activity.
Solanesol mainly occurred in solanaceous crops, such tobacco, tomato, potato, eggplant, and pepper plants. Yan et al. (2015) summarized the resource, derivatives, bioactivities, and medicinal applications of solanesol. Solanesol was widely used as an intermediate for the synthesis of ubiquinone drugs, such as coenzyme Q10 and vitamin K2. Solanesol showed antibacterial, antifungal, antiviral, anticancer, and anti-inflammatory activities. Solanesol derivatives can also be used to treat cardiovascular disease, osteoporosis, and acquired immune deficiency syndrome (AIDS).

Soy isoflavones, such as genistein, daidzein, and glycitein, are important bioactive molecules and considered as antioxidants, antibacterial agents, and free radical-scavengers. Xu et al. (2015) described the potential health benefits of interactions between soy isoflavones and other bioactive components, such as vitamins, trace elements, chemotherapeutics, and phytoestrogens. Raju et al. (2015) summarized the application of LC-MS/MS for the identification and quantification of isoflavonoids reported for various plant extracts and food products. The most suitable and acceptable extraction solvent system for the isoflavonoids is methanol or ethanol in combination with water ranging from 40 to $60 \%$ organic solvent based on the type of tissues and the isoflavonoids to be extracted by different extraction techniques. ESI ionization with Q-TOF MS was the most useful detection system for the characterisation and quantification of the diverse isoflavonoids with molecular insights.

Edible freshwater macrophytes are traditionally used as medicines. Chai et al. (2015) highlighted the phytochemical and pharmacological aspects of the natural products derived from freshwater macrophytes. A promising source of anticancer and antioxidant phytochemicals. The freshwater macrophytes-derived natural products exhibited anticancer and antioxidative bioactivities.

Herbs and spices have been used in retaining and boosting human beauty since time immemorial. Narayanaswamy and Ismail (2015) focused on the cosmetic herbs used in Southeast Asian countries Malaysia, Cambodia, Laos, Myanmar, Thailand, Vietnam, Brunei, East Timor, Indonesia, Philippines and Singapore. Many herbs have been evaluated for their cosmetic potentials such as anti-aging, anti-acne, melanogenic and anti-tyrosinase activities. Labisia 
pumila (Kacip fatimah) and Ficus deltoidea (Mas cotek) are proposed to be clinically studied for their safety in cosmetic application aspects. The traditional herbs including Allium sativum, Aloe vera, Centella asiatica, Curcuma longa, Hibiscus rosa-sinensis, Lawsonia inermis and Tamarindus indica L. were classified as need of special mention.

Brick dark tea is a unique brick formed tea compressed from the older, coarse and rough leaves and branches of Camellia sinensis var. sinensis and $C$. sinensis var. assamica in China. Zheng et al. (2015) reviewed and discussed the modern manufacturing techniques about different kinds of brick dark tea and their influences on the chemical constituents.

Camptothecin is a kind of terpene indole alkaloid which was firstly isolated from native Chinese happy tree Camptotheca acuminata and shows anti-tumor activity. The camptothecin derivatives, irinotecan and topotecan, have been approved by FDA for the treatment of various cancers throughout the world. $\mathrm{Hu}$ et al. (2015) focused on different human malignancies with demonstrated clinical efficacy of camptothecin analogues. The modification of different rings of camptothecin will significantly improve its pharmacokinetics and toxicity profiles. Among them, topotecan, irinotecan and belotecan were proved to be the three most effective camptothecin analogues in the treatment of various malignancies. Kai et al. (2015) summarized the recent advances in the biosynthesis pathway, molecular regulations and various biotechnological approaches for improving the production of camptothecin. Biotechnological approaches such as plant cell suspension, hairy root and endophytic fungi have provided effective platforms to produce camptothecin. Transgenic hairy root culture have revolutionized the role of tissue culture of plants in the production of secondary metabolites. Matveeva et al. (2015) summarized the influence of agrobacterium oncogenes on secondary metabolites of plants.

Lotus seeds are currently the oldest known plant seeds, and contain many functional ingredients. Lotus seeds show prospective application in function food area and traditional medicine research. Zhang et al. (2015) reviewed the nutrition composition, physiological functions and processing methods of lotus seeds and described their impact on nutrient preservation. Proteins and carbohydrates are the main nutrients of lotus seeds. Low fat content and good proportion of amino acids confer to lotus seeds unique nutritional values. The ingredients from lotus seeds exhibited antioxidant activity, hypoglycemic, immunomodulatory, antibacterial, anti-inflammatory, analgesic activities, and gastrointestinal regulation.

Saussurea medusa Maxim (snow lotus) is an important traditional herbal medicine with a broad spectrum of therapeutic efficacies. Fan et al. (2015) summarized the traditional uses and current knowledge concerning the botany, phytochemistry, pharmacological effects, toxicology studies and clinical applications of snow lotus. More than 70 phytochemicals have been isolated and identified, including apigenin, quercetin, rutin, arctigenin, saussureoside A, and saussureoside B. Modern pharmacological studies have found that snow lotus has anti-inflammatory, analgesic, antifungal, antitumor, antioxidant, and antifatigue effects. This plant also benefits the uterus, cardiovascular system, intestines, tracheal smooth muscle, and immune system.

Finally yet importantly, I am thankful to Editor-inChief Prof. Dr. Robert Verpoorte (Institute of Biology Leiden, The Netherlands) for giving me a chance to prepare this special issue compilation as guest editor.

\section{References}

Andrae-Marobela K, Ghislain FW, Okatch H, Majinda R (2013) Polyphenols: a diverse class of multi-target anti-HIV-1 agents. Curr Drug Metab 7:392

Cespedes CL, Aqueveque PM, Avila JG, Alarcon J, Kubo I (2015) New advances in chemical defenses of plants: researches in calceolariaceae. Phytochem Rev. doi:10.1007/ s11101-014-9392-y

Chai T-T, Ooh K-F, Quah Y, Wong F-C (2015) Edible freshwater macrophytes: a source of anticancer and antioxidative natural products-a mini-review. Phytochem Rev. doi:10.1007/s11101-015-9399-Z

Fan JY, Chen HB, Zhu L, Zhao ZZ, Yi T (2015) Saussurea medusa, source of the medicinal herb Snow Lotus: a review of its botany, phytochemistry, pharmacology and toxicology. Phytochem Rev. doi:10.1007/s11101-015-9408-2

Georgiev MI (2013) Coming back to nature: plants as a vital source of pharmaceutically important metabolites-Part II A. Curr Med Chem 20:851

Georgiev MI (2014) Natural products utilization. Phytochem Rev 13:339

Hu GH, Zekria D, Cai X, Ni XL (2015) Current status of CPT and its analogues in the treatment of malignancies. Phytochem Rev. doi:10.1007/s11101-015-9397-1

Kai GY, Wu C, Gen LY, Zhang LQ, Cui LJ, Ni XL (2015) Biosynthesis and biotechnological production of anti-cancer drug Camptothecin. Phytochem Rev. doi:10.1007/ s11101-015-9405-5 
Lanzotti V (2014) Drugs based on natural compounds: recent achievements and future perspectives. Phytochem Rev 13:725

Lanzotti V, Xiao JB (2014) Thematic issue: natural products in cancer prevention and therapy, a selection of topics presented in the 2nd Edition PSE Symposium (Naples, Italy, 25th to 28th of June 2013). Anti Cancer Agents Med Chem $14: 1313$

Matveeva TV, Sokornova SV, Lutova LA (2015) Influence of Agrobacterium oncogenes on secondary methabolism of plants. Phytochem Rev

Narayanaswamy R, Ismail IS (2015) Cosmetic potential of Southeast Asian herbs: an overview. Phytochem Rev. doi:10.1007/s11101-015-9396-2

Raju KSR, Kadian N, Taneja I, Wahajuddin M (2015) Phytochemical analysis of isoflavonoids using liquid chromatography coupled with tandem mass spectrometry. Phytochem Rev. doi:10.1007/s11101-015-9400-x

Xiao JB (2015) Natural polyphenols and diabetes: understanding their mechanism of action. Curr Med Chem 22:2

Xiao JB, Jiang HX (2015) A review on the structure-function relationship aspect of polysaccharides from tea materials. Crit Rev Food Nutr 55:930

Xiao JB, Shao R (2013) Natural products for treatment of Alzheimer's disease and relative diseases: understanding their mechanism of action. Curr Neuropharmacol 11:367

Xiao JB, Muzashvili TS, Georgiev MI (2014) Advance on biotechnology for glycosylation of high-value flavonoids. Biotechnol Adv 32:1145
Xu ML, Liu JB, Zhu CY, Gao Y, Zhao SN, Liu WC, Zhang Y (2015) Interactions between soy isoflavones and other bioactive compounds: a review of their potentially beneficial health effects. Phytochem Rev. doi:10.1007/ s11101-015-9398-0

Yan N, Liu YH, Gong DP, Du YM, Zhang HB, Zhang ZF (2015) Solanesol: a review of its resources, derivatives, bioactivities, medicinal applications, and biosynthesis. Phytochem Rev. doi:10.1007/s11101-015-9393-5

Zeljković SĆ, Maksimović M (2015) Chemical composition and bioactivity of essential oil from Thymus species in Balkan Peninsula. Phytochem Rev. doi:10.1007/s11101-0149378-9

Zhang Y, Lu X, Zeng SX, Huang XH, Guo ZB, Zheng YF, Tian YT, Zheng BD (2015) Nutritional composition, physiological functions and processing of lotus (Nelumbo nucifera Gaertn.) seeds: a review. Phytochem Rev. doi:10. 1007/s11101-015-9401-9

Zheng WJ, Wan XC, Bao GH (2015) Brick dark tea: a review of the manufacture, chemical constituents and bioconversion of the major chemical components during fermentation. Phytochem Rev. doi:10.1007/s11101-015-9402-8

Zhou XL, Wen L, Li ZJ, Zhou YM, Chen YJ, Lu Y (2015) Advance on the benefits of bioactive peptides from buckwheat. Phytochem Rev. doi:10.1007/s11101-014-9390-0 